\title{
DIAGONAL FORMS OF HIGHER DEGREE OVER FUNCTION FIELDS OF $p$-ADIC CURVES
}

\author{
S. PUMPLÜN \\ School of Mathematical Sciences, University of Nottingham, University Park \\ Nottingham NG7 2RD, United Kingdom \\ susanne.pumpluen@nottingham.ac.uk \\ Received (25 May 2018) \\ Accepted (25 June 2019)
}

\begin{abstract}
We investigate diagonal forms of degree $d$ over the function field $F$ of a smooth projective $p$-adic curve: if a form is isotropic over the completion of $F$ with respect to each discrete valuation of $F$, then it is isotropic over certain fields $F_{U}, F_{P}$ and $F_{p}$. These fields appear naturally when applying the methodology of patching; $F$ is the inverse limit of the finite inverse system of fields $\left\{F_{U}, F_{P}, F_{p}\right\}$. Our observations complement some known bounds on the higher $u$-invariant of diagonal forms of degree $d$. $d !$.

We only consider diagonal forms of degree $d$ over fields of characteristic not dividing

Keywords: Forms of higher degree, diagonal forms, function fields, $p$-adic curves, $u$ invariant.
\end{abstract}

Mathematics Subject Classification 2010: 11E76

\section{Introduction}

The fact that Springer's Theorem holds for diagonal forms of higher degree over fields of characteristic not dividing $d$ ! [9] guarantees that on occasion diagonal forms of higher degree defined over function fields behave similarly to quadratic forms. For a survey on the behaviour of (diagonal) forms of higher degree in general the reader is referred to [12].

In this note we consider diagonal forms of degree $d$ over function fields $F=K(X)$ where $X$ is a smooth, projective, geometrically integral curve over $K$ and $K$ is the fraction field of a complete discrete valuation ring with a residue field $k$ of characteristic not dividing $d$ !. Let $v$ be a rank one discrete valuation of $F$, and $F_{v}$ the completion of $F$ with respect to $v$. It was shown by Colliot-Thélène, Parimala and Suresh [2, Theorem 3.1] that a quadratic form which is isotropic over $F_{v}$ for each $v$ is already isotropic over $F$, using the methodology of patching developed by Habater and Hartmann [4], i.e. viewing $F$ as the inverse limit of a finite inverse system of certain fields $\left\{F_{U}, F_{P}, F_{p}\right\}$.

Given a nondegenerate diagonal form $\varphi$ over $F$ of degree $d>2$ and dimension $>2$, it is not clear, however, whether the isotropy of $\varphi$ over $F_{v}$ for each $v$ implies 
that $\varphi$ is isotropic.

Our main result proves that the isotropy of a nondegenerate diagonal form $\varphi$ over $F_{v}$ for each $v$ implies that at least over the field extensions $F_{U}, F_{P}$ and $F_{p}$ of $F$, $\varphi$ is isotropic as well (Theorem 3.1). These fields depend on the choice of the form $\varphi=\left\langle a_{1}, \ldots, a_{n}\right\rangle$, more precisely on the choice of the regular proper model $\mathcal{X}$ (over the complete discrete valuation ring $A$ ) of the curve $X$ over $K$, which depends on $\varphi$ : $\mathcal{X}$ is selected such that there exists a reduced divisor $D$ with strict normal crossings, which contains both the support of the divisor of all the entries $a_{i}, 1 \leq i \leq n$, and the components of the special fibre of $X / A$. Since nondegenerate diagonal forms of degree $d \geq 3$ have finite automorphism groups [6, p. 137], we are not able to apply [5, Theorem 3.7] to conclude that the isotropy of $\varphi$ over the $F_{U}$ 's and $F_{P}$ 's implies that $\varphi$ is also isotropic over $F$, however. This is only possible for $d=2$.

After collecting the terminology and some basic results in Section 1, in particular defining diagonal $u$-invariants of degree $d$ over $k$, we consider diagonal forms of higher degree over valued fields in Section 2 and then study diagonal forms of higher degree over function fields of $p$-adic curves using some of the ideas of [2] in Section 3. Recall that a $p$-adic field is a finite field extension of $\mathbb{Q}_{p}$.

As a consequence of Springer's Theorem for diagonal forms, any diagonal form of degree $d$ and dimension $>d^{3}+1$ over a function field in one variable $F=K(t)$, where $K$ is a $p$-adic field with residue field $k$, $\operatorname{char}(k) \nmid d$ !, is isotropic over $F_{v}$ for every discrete valuation $v$ with residue field either a function field in one variable over $k$ or a finite extension of $K$. Moreover, it is isotropic over $F_{U}$ for each reduced, irreducible component $U \subset Y$ of the complement of $S$ in the special fibre $Y=\mathcal{X} \times{ }_{A} k$ of $X / A$, and isotropic over $F_{P}$ for each $P \in S$ (Corollary 3.4), and thus isotropic over $F_{p}$ for each $p=(U, P)$. Here, $S$ is the inverse image under a finite $A$-morphism $f: \mathcal{X} \rightarrow \mathbb{P}_{A}^{1}$ of the point at infinity of the special fibre $\mathbb{P}_{k}^{1}$.

\section{Preliminaries}

Let $k$ be a field such that $\operatorname{char}(k)$ does not divide $d$ !.

\subsection{Forms of higher degree}

Let $V$ be a finite-dimensional vector space over $k$ of dimension $n$. A d-linear form over $k$ is a $k$-multilinear map $\theta: V \times \cdots \times V \rightarrow k$ ( $d$-copies) which is symmetric, i.e. $\theta\left(v_{1}, \ldots, v_{d}\right)$ is invariant under all permutations of its variables. A form of degree $d$ over $k$ (and of dimension $n$ ) is a map $\varphi: V \rightarrow k$ such that $\varphi(a v)=a^{d} \varphi(v)$ for all $a \in k, v \in V$ and such that the map $\theta: V \times \cdots \times V \rightarrow k$ defined by

$$
\theta\left(v_{1}, \ldots, v_{d}\right)=\frac{1}{d !} \sum_{1 \leq i_{1}<\cdots<i_{l} \leq d}(-1)^{d-l} \varphi\left(v_{i_{1}}+\cdots+v_{i_{l}}\right)
$$

$(1 \leq l \leq d)$ is a $d$-linear form over $k$. By fixing a basis $\left\{e_{1}, \ldots, e_{n}\right\}$ of $V$, any form $\varphi$ of degree $d$ can be viewed as a homogeneous polynomial of degree $d$ in $n=\operatorname{dim} V$ variables $x_{1}, \ldots, x_{n}$ via $\varphi\left(x_{1}, \ldots, x_{n}\right)=\varphi\left(x_{1} e_{1}+\cdots+x_{n} e_{n}\right)$ and, vice versa, any 
homogeneous polynomial of degree $d$ in $n$ variables over $k$ is a form of degree $d$ and dimension $n$ over $k$. Any $d$-linear form $\theta: V \times \cdots \times V \rightarrow k$ induces a form $\varphi: V \rightarrow k$ of degree $d$ via $\varphi(v)=\theta(v, \ldots, v)$. We can hence identify $d$-linear forms and forms of degree $d$.

Two $d$-linear spaces $\left(V_{i}, \theta_{i}\right), i=1,2$, are isomorphic (written $\left(V_{1}, \theta_{1}\right) \cong\left(V_{2}, \theta_{2}\right)$ or just $\left.\theta_{1} \cong \theta_{2}\right)$ if there exists a bijective linear map $f: V_{1} \rightarrow V_{2}$ such that $\theta_{2}\left(f\left(v_{1}\right), \ldots, f\left(v_{d}\right)\right)=\theta_{1}\left(v_{1}, \ldots, v_{d}\right)$ for all $v_{1}, \ldots, v_{d} \in V_{1}$. A $d$-linear space $(V, \theta)$ (or the $d$-linear form $\theta$ ) is nondegenerate if $v=0$ is the only vector such that $\theta\left(v, v_{2}, \ldots, v_{d}\right)=0$ for all $v_{i} \in V$. A form of degree $d$ is called nondegenerate if its associated $d$-linear form is nondegenerate. A form $\varphi$ over $k$ is called anisotropic, if it does not have any non-trivial zeroes, otherwise it is called isotropic.

The orthogonal sum $\left(V_{1}, \theta_{1}\right) \perp\left(V_{2}, \theta_{2}\right)$ of two $d$-linear spaces $\left(V_{i}, \theta_{i}\right), i=1,2$, is the $k$-vector space $V_{1} \oplus V_{2}$ together with the $d$-linear form

$$
\left(\theta_{1} \perp \theta_{2}\right)\left(u_{1}+v_{1}, \ldots, u_{d}+v_{d}\right)=\theta_{1}\left(u_{1}, \ldots, u_{d}\right)+\theta_{2}\left(v_{1}, \ldots, v_{d}\right)
$$

$\left(u_{i} \in V_{1}, v_{i} \in V_{2}\right)[7]$.

A $d$-linear space $(V, \theta)$ is called decomposable if $(V, \theta) \cong\left(V, \theta_{1}\right) \perp\left(V, \theta_{2}\right)$ for two non-zero $d$-linear spaces $\left(V, \theta_{i}\right), i=1,2$. If $\varphi$ is represented by the homogeneous polynomial $a_{1} x_{1}^{d}+\ldots+a_{m} x_{m}^{d}\left(a_{i} \in k^{\times}\right)$we use the notation $\varphi=\left\langle a_{1}, \ldots, a_{n}\right\rangle$ and call $\varphi$ diagonal. A diagonal form $\varphi=\left\langle a_{1}, \ldots, a_{n}\right\rangle$ over $k$ is nondegenerate if and only if $a_{i} \in k^{\times}$for all $1 \leq i \leq n$.

If $d \geq 3, a_{i}, b_{j} \in k^{\times}$, then $\left\langle a_{1}, \ldots, a_{n}\right\rangle \cong\left\langle b_{1}, \ldots, b_{n}\right\rangle$ if and only if there is a permutation $\pi \in S_{n}$ such that $\left\langle b_{i}\right\rangle \cong\left\langle a_{\pi(i)}\right\rangle$ for every $i$. This is a special case of $[6$, Theorem 2.3].

Note that for quadratic forms $(d=2)$, the automorphism group of $\varphi$ is infinite, whereas for $d \geq 3$, the automorphism group of $\varphi$ usually is finite, for instance if $\varphi$ is is nonsingular in the sense of algebraic geometry [13]. In particular, nondegenerate diagonal forms of degree $d \geq 3$ have finite automorphism groups [6, p. 137], which creates a problem when trying to imitate patching arguments as it is not possible to apply [5, Theorem 3.7].

\subsection{Higher degree u-invariants}

The $u$-invariant (of degree $d$ ) of $k$ is defined as $u(d, k)=\sup \left\{\operatorname{dim}_{k} \varphi\right\}$, where $\varphi$ ranges over all the anisotropic forms of degree $d$ over $k$. The diagonal $u$-invariant (of degree $d$ ) of $k$ is defined as $u_{\text {diag }}(d, k)=\sup \{\operatorname{dim} \varphi\}$, where $\varphi$ ranges over all the anisotropic diagonal forms over $k$.

Thus the diagonal $u$-invariant $u_{\text {diag }}(d, k)$ is the smallest integer $n$ such that all diagonal forms of degree $d$ over $k$ of dimension greater than $n$ are isotropic, and the $u$-invariant $u(d, k)$ is the smallest integer $n$ such that all forms of degree $d$ over $k$ of dimension greater than $n$ are isotropic. Obviously, $u_{\text {diag }}(d, k) \leq u(d, k)$. If $u=u(d, k)$ then each anisotropic form of degree $d$ over $k$ of dimension $u$ is universal. If $u=u_{\text {diag }}(d, k)$ then each diagonal anisotropic form of degree $d$ over $k$ 
of dimension $u$ is universal. We have

$$
u_{\text {diag }}(d, k) \leq \min \{n \mid \text { all forms of degree } d \text { over } k \text { of dimension } \geq n \text { are universal }\}
$$

with the understanding that the "minimum" of an empty set of integers is $\infty$, cf. $[12]$. For $d=2, u_{\text {diag }}(d, k)=u(d, k)$ is the $u$-invariant of quadratic forms.

For an algebraically closed field $k,\left|k^{\times} / k^{\times d}\right|=1$ and hence $u_{\text {diag }}(d, k)=$ $u(d, k)=1$. For a formally real field $k$, the diagonal $u$-invariant is infinite for even $d$ : since $-1 \notin \sum k^{2}$, also $-1 \notin \sum k^{d}$ for any even $d$. Thus the form $m \times\langle 1\rangle$ of degree $d$ is anisotropic for each integer $m$, implying $u_{\text {diag }}(d, k)=u(d, k)=\infty$.

The strong diagonal $u$-invariant of degree $d$ of $k$, denoted $u_{\text {diag, } s}(d, k)$, is the smallest real number $n$ such that

(1) every finite field extension $E / k$ satisfies $u_{\text {diag }}(d, E) \leq n$, and

(2) every finitely generated field extension $E / k$ of transcendence degree one satisfies $u_{\text {diag }}(d, E) \leq d n$.

If these $u$-invariants are arbitrarily large, put $u_{\text {diag, } s}(d, k)=\infty$.

Analogously as observed in [5] for $d=2, u_{\text {diag, } s}(d, k) \leq n$ if and only if every finitely generated field extension $E / k$ of transcendence degree $l \geq 1$ satisfies $u_{\text {diag }}(d, k) \leq d^{l} n$. Thus if $u_{\text {diag }, s}(d, k)$ is finite, it is at least 1 and lies in $\frac{1}{d} \mathbb{N}$.

\section{3. $C_{r}^{0}$ fields}

Let $r \geq 1$ be an integer. A field $F$ is a $C_{r}$-field if for all $d \geq 1$ and $n>d^{r}$, every homogeneous form of degree $d$ in $n$ variables over $F$ has a non-trivial solution in $F$. In particular, then $F$ satisfies $u(d, F) \leq d^{r}$. Moreover, every finite extension of $F$ is a $C_{r}$-field, and every one-variable function field over $F$ a $C_{r+1}$-field [14, II.4.5]. Hence $u_{\text {diag, } s}(d, F) \leq d^{r}$ for a $C_{r}$-field $F$.

A field $F$ is a $C_{r}^{0}$-field if the following holds: For any finite field extension $F^{\prime}$ of $F$ and any integers $d \geq 1$ and $n>d^{r}$, for any homogeneous form over $F^{\prime}$ of degree $d$ in $n$ variables, the greatest common divisor of the degrees of finite field extensions $F^{\prime \prime} / F^{\prime}$ over which the form acquires a nontrivial zero is one. This amounts to requiring that the $F^{\prime}$-hypersurface defined by the form has a zero-cycle of degree 1 over $F^{\prime}$.

Assume $\operatorname{char}(F)=0$. For each prime $l$, let $F_{l}$ be the fixed field of a pro- $l$-Sylow subgroup of the absolute Galois group of $F$. Any finite subextension of $F_{l} / F$ is of degree coprime to $l$. The field $F$ is $C_{r}^{0}$ if and only if each of the fields $F_{l}$ is $C_{r}$. A finite field extension of a $C_{r}^{0}$-field is $C_{r}^{0}$. If $F$ is $C_{r}^{0}$ then a function field $E=F\left(x_{1}, \ldots, x_{s}\right)$ in $s$ indeterminates $x_{1}, \ldots, x_{s}$ over $F$ is $C_{r+s}^{0}[2,2.1]$.

It is not known if $p$-adic fields have the $C_{2}^{0}$-property.

Remark 1.1. Assume that $p$-adic fields have the $C_{2}^{0}$-property. Let $K(X)$ be any function field of transcendence degree $r$ over a $p$-adic field $K$ (here we do not need to assume $p \neq 2,3)$. Suppose that there is $\ell \neq 2$ such that there exists a finite subextension of $K_{\ell}(X) / K(X)$ of degree 2. Then any cubic form over $K(X)$ in 
strictly more than $3^{2+r}$ variables has a nontrivial zero: If the $p$-adic field $K$ is $C_{3}^{0}$, then the function field $E=K(X)$ in $r$ variables over $K$ is $C_{3+r}^{0}$ [2, Lemma 2.1]. Thus a cubic form over $E=K(X)$ in strictly more than $3^{2+r}$ has a nontrivial zero in each of the fields $K_{\ell}(X), l$ a prime, hence in a finite extension of $K(X)$ of degree coprime to $\ell$, for each $\ell$ prime. Pick $\ell \neq 2$, then $\left[K_{\ell}(X): K(X)\right]$ is even. Moreover, pick $l \neq 2$ such that there exists a finite subextension of $E_{\ell} / K(X)$ of degree 2 then the cubic form has a zero over it. By Springer's Theorem for cubic forms and their behaviour under quadratic field extensions $[8, \mathrm{VII}]$, thus the cubic form has a nontrivial zero in $K(X)$. This is the analogue of [2, Proposition 2.2].

\section{Diagonal forms over Henselian valued fields}

\section{1.}

Let $K$ be a valued field with valuation $v$, valuation $\operatorname{ring} R$ and maximal ideal $m$. Let $\Gamma$ be the value group. Assume that $d$ ! is not divisible by the characteristic of the residue field $k=R / m$. For $u \in R$, denote by $\bar{u}$ the image of $u$ in $k$. For a polynomial $f \in R[X], f=a_{n} x^{n}+\cdots+a_{1} x+a_{0}$, define the polynomial $\bar{f}=\bar{a}_{n} x^{n}+\cdots+\bar{a}_{1} x+\bar{a}_{0}$ over $k$. If $\varphi=\left\langle a_{1}, \ldots, a_{n}\right\rangle$ is a nondegenerate diagonal form with entries $a_{i} \in R$, define the diagonal form $\bar{\varphi}=\left\langle\bar{a}_{1}, \ldots, \bar{a}_{n}\right\rangle$ over $k . \varphi$ is called a unit form, if $\bar{\varphi}$ is nondegenerate. Choose a set $\left\{\pi_{\gamma} \in R \mid \gamma \in I\right\}$ such that the values of the $\pi_{\gamma}$ 's represent the distinct cosets in $\Gamma / d \Gamma$. We may decompose a diagonal form $\varphi$ as $\varphi=\perp \varphi_{\gamma}^{\prime}$ by taking $\varphi_{\gamma}^{\prime}$ to be the diagonal form whose entries comprise all $a_{i}$ with $v\left(a_{i}\right)=v\left(\pi_{\gamma}\right) \bmod d \Gamma$. By altering the slots by $d$-powers if necessary, we may then write $\varphi_{\gamma}^{\prime}=\pi_{\gamma} \varphi_{\gamma}$ with each $\varphi_{\gamma}$ a diagonal unit form. There are only finitely many non-trivial $\varphi_{\gamma}$ [9]. If $\Gamma=\mathbb{Z}$, the set $\left\{\pi_{\gamma} \mid \gamma \in I\right\}$ can be chosen to be $\left\{\pi^{i} \mid i=0, \ldots, d-1\right\}$ and $|\Gamma / d \Gamma|=d$ is finite.

If $R$ satisfies Hensel's Lemma then $(K, v)$ is called a Henselian valued field and $R$ a Henselian valuation ring. Every complete discretely valued field is Henselian.

Let $\varphi$ be a diagonal form over a Henselian valued field $(K, v)$. Write $\varphi=\pi_{1} \varphi_{1} \perp$ $\cdots \perp \pi_{r} \varphi_{r}$ with each $\varphi_{i}$ a diagonal unit form and the $\pi_{i}$ having distinct values in $\Gamma / d \Gamma$. Then $\varphi$ is isotropic if and only if some $\overline{\varphi_{i}}$ is isotropic [9, Proposition 3.1]. This is because for a diagonal unit form $\varphi$ over a Henselian valued field $(K, v), \varphi$ is isotropic if and only if $\bar{\varphi}$ is isotropic [9, Lemma 2.3].

Theorem 2.1. ([9] or [12, Theorem 4, Corollary 2]) Suppose that char $(k) \nmid d$ !. (i) Let $(K, v)$ be a Henselian valued field. Then $u_{\text {diag }}(d, K)=|\Gamma / d \Gamma| u_{\text {diag }}(d, k)$.

(ii) Let $(K, v)$ be a Henselian valued field. If every diagonal form of degree $d$ of dimension $n+1$ over $k$ is isotropic, then every diagonal form of degree $d$ and dimension $d n+1$ over $K$ is isotropic. If $k$ has an anisotropic form of degree $d$ and dimension $n$, then $K$ has an anisotropic form of degree $d$ and dimension $d n$.

(iii) Let $K$ be a discretely valued field. Then $u_{\text {diag }}(d, K) \geq d u_{\text {diag }}(d, k)$.

(iv) Let $F$ be a field extension of finite type over $k$ of transcendence degree $n$. Then $u_{\text {diag }}(d, F) \geq d^{n} u_{\text {diag }}\left(d, k^{\prime}\right)$ for a suitable finite field extension $k^{\prime} / k$. 
The (in)equalities in (i), (iii), (iv) also hold when the values are infinite.

For $d=2$, (ii) is Springer's Theorem for quadratic forms over Henselian valued fields [15]. Springer's Theorem does not hold for non-diagonal forms of higher degree than $2[9,2.7]$. Theorem 2.1 is a major ingredient in our proofs, for instance we can show:

Proposition 2.2. Let $A$ be a discrete valuation ring with fraction field $K$ and residue field $k$ such that $\operatorname{char}(k) \nmid d$ !.

(i) $u_{\text {diag }}(d, K) \geq d u_{\text {diag }}(d, k)$ and $u_{\text {diag }, s}(d, K) \geq d u_{\text {diag, },}(d, k)$.

(ii) If $A$ is Henselian then $u_{\text {diag }}(d, K)=d u_{\text {diag }}(d, k)$.

(iii) If $A$ is a complete discrete valuation ring then every finite extension of $K$ has diagonal u-invariant at most $d u_{\text {diag }}(d, k)$.

The first assertions of (i) as well as (ii) and (iii) follow from Theorem 2.1. The proof of the second claim in (i) is analogous to the one of [5, 4.9], employing Theorem 2.1 instead of Springer's Theorem.

\section{2 .}

A field $K$ is called an $m$-local field with residue field $k$ if there is a sequence of fields $k_{0}, \ldots, k_{m}$ with $k_{0}=k$ and $k_{m}=K$, and such that $k_{i}$ is the fraction field of an excellent Henselian discrete valuation ring with residue field $k_{i-1}$ for $i=1, \ldots, m$. Recall that a discrete valuation ring $R$ is called excellent, if the field extension $\widehat{K} / K$ is separable, where $\widehat{K}$ denotes the quotient field of $R$ and $K$ is its completion. (This condition is trivially satisfied if $K$ has characteristic 0 or $R$ is complete.)

Proposition 2.2 implies (compare the next two results with [5, Corollary 4.13, 4.14] for quadratic forms):

Corollary 2.3. Suppose that $K$ is an $m$-local field whose residue field $k$ is a $C_{r}$ field with $\operatorname{char}(k) \nmid d$ !. Let $F$ be a function field over $K$ in one variable.

(i) $u_{\text {diag }}(d, k)=u_{\text {diag }, s}(d, k)=d^{r}$ and $u_{\text {diag }}(d, K)=d^{r+m}$.

Moreover, if some normal $K$-curve with function field $F$ has a $K$-point, then $u_{\text {diag }}(d, F) \geq d^{r+m+1}$.

(ii) If $u_{\text {diag }}\left(d, k^{\prime}\right)=d^{r}$ for every finite extension $k^{\prime} / k$, then $u_{\text {diag }}(d, F) \geq d^{r+m+1}$.

Proof. (i) Since $k$ is a $C_{r}$-field, $u_{\text {diag }}(d, k)=d^{r}$, thus $u_{\text {diag }}(d, k) \leq u_{\text {diag }, s}(d, k) \leq$ $d^{r}$ and the first two equations follow. Applying Proposition 2.2 and induction yields that $u_{\text {diag }}(d, K) \geq d^{m} u_{\text {diag }}(d, k)=d^{r+m}$. Let $X$ be a normal $K$-curve with function field $F$ and let $\xi$ be a $K$-point on $X$. The local ring at $\xi$ has fraction field $F$ and residue field $K$. So Proposition 2.2 implies that $u_{\text {diag }}(d, K)=d^{m} u_{\text {diag }}(d, k)$ and $u_{\text {diag }}(d, F) \geq d u_{\text {diag }}(d, K)=d^{r+m+1}$

(ii) Choose a normal or equivalently a regular $K$-curve $X$ with function field $F$, and a closed point $\xi$ on $X$. Let $R$ be the local ring of $X$ at $\xi$ with residue field $\kappa(\xi)$. Then the fraction field of $R$ is $F$, and $\kappa(\xi)$ is a finite extension of $K$. Hence $\kappa(\xi)$ 
is an $m$-local field whose residue field $k^{\prime}$ is a finite extension of $k$. By assumption, $u_{\text {diag }}\left(d, k^{\prime}\right)=d^{r}$ and $k^{\prime}$ is a $C_{r}$-field since $k$ is. So applying part (ii) to $k^{\prime}$ and $\kappa(\xi)$, it follows that $u_{\text {diag }}(d, \kappa(\xi)) \geq d^{r+m}$. Proposition 2.2 yields $u_{\text {diag }}(d, F) \geq d^{r+m+1} \square$

Corollary 2.4. (i) Let $F$ be a one-variable function field over an $m$-local field $K$ with residue field $k$ such that $\operatorname{char}(k) \nmid d$ ! and $k$ is algebraically closed. Then $u_{\text {diag }}(d, F) \geq d^{m+1}$.

(ii) If $k$ is a finite field and $u_{\text {diag }}(d, k)=d^{r}$ with $r \in\{0,1\}$, then $u_{\text {diag }}(d, k)=$ $u_{\text {diag }, s}(d, k)=d^{r}$ and $u_{\text {diag }}(d, K) \geq d^{r+m}$. Moreover, if some normal $K$-curve with function field $F$ has a $K$-point, then $u_{\text {diag }}(d, F) \geq d^{r+m+1}$.

Proof. (i) This is a special case of Corollary 2.3 using that an algebraically closed field $k$ is $C_{0}$, satisfies $u_{\text {diag }}(d, k)=1$, and has no non-trivial finite extensions.

(ii) A finite field is $C_{1}[14$, II.3.3(a) $]$, hence $u_{\text {diag }}(d, k) \leq d$. Here $u_{\text {diag }}(d, k)=$ $u_{\text {diag, } s}(d, k)=d^{r}$ and Corollary 2.3 yields the assumption.

In general, for any finite field $k=\mathbb{F}_{q}$ we obviously do not have $u_{\text {diag }}(d, k)=d^{r}$, $r \in\{0,1\}$ : for instance, if $-1 \in \mathbb{F}_{q}^{\times d}$ and $d \geq 4$ then $u_{\text {diag }}\left(d, \mathbb{F}_{q}\right) \leq d-1$ by [10]. Or, if $d^{*}=\operatorname{gcd}(d, q-1)$, then $u_{\text {diag }}\left(d, \mathbb{F}_{q}\right) \leq d^{*}$. This implies that $u_{\text {diag }}\left(d, \mathbb{F}_{q}\right)=1$, if $d$ is relatively prime to $q-1$ and that for $q>\left(d^{*}-1\right)^{4}, u_{\text {diag }}\left(d, \mathbb{F}_{q}\right)=2[12,5.1]$.

\section{The behaviour of diagonal forms of higher degree over function fields of $p$-adic curves}

Whenever we write 'discrete valuation ring' and 'discrete valuation' we mean a discrete valuation ring of rank one and a valuation with value group $\mathbb{Z}$.

\section{1 .}

Let $A$ be a complete discrete valuation ring with fraction field $K$ and residue field $k$ with $\operatorname{char}(k) \nmid d$ !. Let $X$ be a smooth, projective, geometrically integral curve over $K$ and $F=K(X)$ be the function field of $X$. Let $t$ denote a uniformizing parameter for $A$. For each (rank one) discrete valuation $v$ of $F$, let $F_{v}$ denote the completion of $F$ with respect to $v$.

We will adapt some ideas from [2] to diagonal forms of higher degree: take a nondegenerate form $\varphi=\left\langle a_{1}, \ldots, a_{n}\right\rangle$ of degree $d$ over $F$. Then choose a regular proper model $\mathcal{X} / A$ of $X / K$, such that there exists a reduced divisor $D$ with strict normal crossings which contains both the support of the divisor of all the entries $a_{i}$, $1 \leq i \leq n$, and the components of the special fibre of $X / A$. (Note that this implies that the regular proper model $\mathcal{X} / A$ depends on the form $\varphi$, and thus so do $Y, Y_{i}$, $S_{0}, S, F_{P}, F_{U}, \ldots$ as defined in the following.)

Let $Y=\mathcal{X} \times{ }_{A} k$ be the special fibre of $X / A$. Let $x_{i}$ be the generic point of an irreducible component $Y_{i}$ of $Y$. Then there is an affine Zariski neighbourhood $W_{i} \subset \mathcal{X}$ of $x_{i}$, such that the restriction of $Y_{i}$ to $W_{i}$ is a principal divisor. Let $S_{0}$ 
be a finite set of closed points of $Y$ containing all singular points of $D$, and all the points that lie on some $Y_{i}$, but not in the corresponding $W_{i}$.

Choose a finite $A$-morphism $f: \mathcal{X} \rightarrow \mathbb{P}_{A}^{1}$ as in [4, Proposition 6.6]. Let $S$ be the inverse image under $f$ of the point at infinity of the special fibre $\mathbb{P}_{k}^{1}$. Then the set $S_{0}$ is contained in $S$. All the intersection points of two components $Y_{i}$ are in $S$. Each component $Y_{i}$ contains at least one point of $S$. Let $U \subset Y$ run through the reduced irreducible components of the complement of $S$ in $Y$. Then each $U$ is a regular affine irreducible curve over $k$ and we define $k[U]$ to be its ring of regular functions and $k(U)$ to be its fraction field. $k[U]$ is a Dedekind domain and $U=\operatorname{Spec} k[U]$. Each $U$ is contained in an open affine subscheme $\operatorname{Spec} R^{U}$ of $\mathcal{X}$ and is a principal effective divisor in $\operatorname{Spec} R^{U}$. Moreover, $R_{U}$ is the ring of elements in $F$ which are regular on $U$ and also a regular ring, since it is the direct limit of regular rings. The $\operatorname{ring} R_{U}$ is a localisation of $R^{U}$ and so $U$ is a principal effective divisor on Spec $R_{U}$ given by the vanishing of an element $s \in R_{U}$. The $t$-adic completion $\widehat{R}_{U}$ of $R_{U}$ is a domain and coincides with the $s$-adic completion of $R_{U}$, since $t=u s^{r}$ for some integer $r \geq 1$ and a unit $u \in R_{U}^{\times}$. By definition, $F_{U}$ is the field of fractions of $\widehat{R}_{U}$. We have $k[U]=R_{U} / s=\widehat{R}_{U} / s$. For $P \in S$, the completion $\widehat{R}_{P}$ of the local ring $R_{P}$ of $\mathcal{X}$ at $P$ is a domain and $F_{P}$ is the field of fractions of $\widehat{R}_{P}$. Let $p=(U, P)$ be a pair with $P \in S$ in the closure of an irreducible component $U$ of the complement of $S$ in $Y$. Then let $R_{p}$ be the complete discrete valuation ring which is the completion of the localisation of $\widehat{R}_{P}$ at the height one prime ideal corresponding to $U$. Then $F_{p}$ is the field of fractions of $\widehat{R}_{p}$ and $F$ is the inverse limit of the finite inverse system of fields $\left\{F_{U}, F_{P}, F_{p}\right\}$ by [4, Proposition 6.3].

The following can be seen as a weak generalization of [2, Theorem 3.1] to diagonal forms of higher degree. Here we are not able to conclude that under the given assumptions, $\varphi$ is isotropic over $F$, only over the $F_{U}$ 's and $F_{P}$ 's:

Theorem 3.1. Let $\varphi$ be a nondegenerate diagonal form of degreed over $F$. If $\varphi$ is isotropic over the completion $F_{v}$ of $F$ with respect to each discrete valuation $v$ of $F$ with residue field either a function field in one variable over $k$ or a finite extension of $K$, then:

(i) $\varphi$ is isotropic over $F_{U}$ for each reduced irreducible component $U \subset Y$ of the complement of $S$ in $Y$,

(ii) $\varphi$ is isotropic over $F_{P}$ for each $P \in S$.

Proof. Suppose $\varphi=\left\langle a_{1}, \ldots, a_{n}\right\rangle$.

(i) Each entry $a_{i}$ of $\varphi$ is supported only along $U$ in $\operatorname{Spec} R_{U}$, thus has the form $u s^{j}$ where $u \in R_{U}^{\times}$. We sort the entries $a_{i}=u_{i} s^{j}$ by the power $j$ of $s$ and use them to define new diagonal forms $\rho_{j}$ which have all the $u_{i}$ 's belonging to those $a_{i}$ where $s$ occurred in the $j$ th power as their diagonal entries. Hence $\varphi$ is isomorphic to the diagonal form

$$
\rho_{0} \perp s \rho_{1} \perp \cdots \perp s^{d-1} \rho_{d-1}
$$

over $F$, where the $\rho_{i}$ are nondegenerate diagonal forms of degree $d$ over $R_{U}$. Note 
that if for some $j \in\{0,1, \ldots, d-1\}$ there is no $a_{i}$ with $a_{i}=u_{i} s^{j}$, then there is no corresponding form $\rho_{j}$ and a $\rho_{j}$ does not appear as a component in the sum.

By hypothesis, $\varphi$ is isotropic over the field of fractions of the completed local ring of $\mathcal{X}$ at the generic point of $U$. By Theorem 2.1, this implies that the image of at least one of the forms $\rho_{0}, \rho_{1}$ or $\rho_{d-1}$ under the composite homomorphism $R_{U} \rightarrow k[U] \rightarrow k(U)$ is isotropic over $k(U)$. Since the residue characteristic $p$ does not divide $d$ !, the forms $\rho_{0}, \rho_{1}, \ldots, \rho_{d-1}$ define a smooth projective variety over $R_{U}$. In particular, all of them define a smooth variety over $k[U]$. Since $k[U]$ is a Dedekind domain, if such a projective variety has a point over $k(U)$, it has a point over $k[U]$. Since the variety is smooth over $R_{U}$, a $k[U]$-point lifts to an $\widehat{R}_{U}$-point (cf. the discussion after [5, Lemma 4.5]). Thus $\varphi$ has a nontrivial zero over $F_{U}$.

(ii) Let $P \in S$. The local ring $R_{P}$ of $\mathcal{X}$ at $P$ is regular. Its maximal ideal is generated by two elements $(x, y)$ with the property that any $a_{i}$ is the product of a unit, a power of $x$ and a power of $y$. Thus over $F$, the fraction field of $R_{P}, \varphi$ is isomorphic to

$$
\begin{gathered}
\varphi_{1} \perp x \varphi_{2} \perp y \varphi_{3} \perp x y \varphi_{4} \perp x^{2} \varphi_{5} \perp y^{2} \varphi_{6} \perp x^{2} y^{2} \varphi_{7} \perp x^{2} y \varphi_{8} \perp x y^{2} \varphi_{9} \perp \\
\cdots \perp x^{d-1} y^{d-1} \varphi_{d^{2}},
\end{gathered}
$$

where each $\varphi_{i}$ is a nondegenerate diagonal form over $R_{P}$. Let $R_{y}$ be the localization of $R_{P}$ at the prime ideal $(y) . R_{y}$ is a discrete valuation ring with fraction field $F$. The residue field $E$ of $R_{y}$ is the field of fractions of the discrete valuation ring $R_{P} /(y)$. By hypothesis, the form

$$
\begin{gathered}
\left(\varphi_{1} \perp x \varphi_{2} \perp x^{2} \varphi_{5} \perp \ldots\right) \perp y\left(\varphi_{3} \perp x \varphi_{4} \perp \ldots\right) \\
\perp y^{2}\left(\varphi_{6} \perp \ldots\right) \perp \cdots \perp y^{d-1}\left(\cdots \perp x^{d-1} \varphi_{d^{2}}\right)
\end{gathered}
$$

is isotropic over the field of fractions of the completion of $R_{y}$. By Theorem 2.1, the reduction of one of the forms

$$
\left(\varphi_{1} \perp x \varphi_{2} \perp x^{2} \varphi_{5} \perp \ldots\right),\left(\varphi_{3} \perp x \varphi_{4} \perp \ldots\right), \cdots,
$$

is isotropic over $E$. Since $x$ is a uniformizing parameter for $R_{P} /(y)$, by Theorem 2.1 this implies that over the residue field of $R_{P} /(y)$, the reduction of one of the forms $\varphi_{1}, \varphi_{2}, \varphi_{3} \ldots, \varphi_{d^{2}}$ is isotropic. But then one of these forms is isotropic over $\widehat{R}_{P}$, hence over the field $F_{P}$ which is the fraction field of $\widehat{R}_{P}$.

Remark 3.2. (i) In the proof of Theorem 3.1, one of the forms is isotropic over $\widehat{R}_{P}$, and since $R_{p}$ is the complete discrete valuation ring which is the completion of the localisation of $\widehat{R}_{P}$ at the height one prime ideal corresponding to $U$ when $p=(U, P)$, this form is also isotropic over $R_{p}$ and therefore over the field of fractions $F_{p}$ of $\widehat{R}_{p}$. This implies that if $\varphi$ is isotropic over the completion of $F$ with respect to each discrete valuation of $F$, then $\varphi$ is isotropic over $F_{U}$ for each reduced irreducible component $U \subset Y$ of the complement of $S$ in $Y$, over $F_{P}$ for each $P \in S$ and over $R_{p}$ for each $p=(U, P)$. Since $F$ is the inverse limit of the finite inverse system of 
fields $\left\{F_{U}, F_{P}, F_{p}\right\}, \varphi$ is isotropic over all overfields used in the inverse limit.

(ii) The discrete valuation rings used in the above proof are the local rings at a point of codimension 1 on a suitable regular proper model $\mathcal{X}$ of $X$ determined by the choice of $\varphi$ (analogously as noted in [2, Remark 3.2]).

Given a nondegenerate diagonal form $\varphi$ of degree $d$ and dimension greater than two over $F$, it is not clear whether the isotropy of $\varphi$ over $F_{v}$ for each $v$ (respectively, of $\varphi$ over all $F_{U}, F_{P}$ and $F_{p}$ ) implies that $\varphi$ is isotropic (the fact that $\operatorname{dim} \varphi>2$ is necessary: it is easy to adjust the example in [2, Appendix] to two-dimensional diagonal forms of even degree).

Corollary 3.3. Let $r \geq 1$ be an integer and $d \geq 3$. Assume that any diagonal form in strictly more than $d r$ variables over any function field in one variable over $k$ is isotropic. Then:

(i) Any diagonal form of degree $d$ and dimension $>d^{2} r$ over the function field $F=K(X)$ of a curve $X / K$ is isotropic over $F_{v}$, for every discrete valuation $v$ with residue field either a function field in one variable over $k$ or a finite extension of $K$.

(ii) Any diagonal form of degree $d$ and dimension $>d^{2} r$ over the function field $F=$ $K(X)$ of a curve $X / K$ is isotropic over $F_{U}$ for each reduced, irreducible component $U \subset Y$ of the complement of $S$ in $Y$ and is isotropic over $F_{P}$ for each $P \in S$.

Note that $Y$ and $S$ depend on $\varphi$.

Proof. (i) Let $L$ be a finite field extension of $K$. This is a complete discretely valued field with residue field a finite extension $\ell$ of $k$. The assumption made on diagonal forms of degree $d$ over functions fields in one variable over $k$, in particular diagonal forms of degree $d$ over the field $\ell(t)$, and Theorem 2.1 applied to $\ell((t))$ show that any diagonal form of dimension $>r$ over $\ell$ has a zero. A second application of Theorem 2.1 yields that any diagonal form of degree $d$ of dimension $>d r$ over $L$ is isotropic. Let $\varphi$ be a diagonal form of dimension $n$ over $F$ with $n>d^{2} r$. By the assumption and Theorem 2.1, $\varphi$ is isotropic over $F_{v}$ for every discrete valuation $v$ with residue field either a function field in one variable over $k$ or a finite extension of $K$.

(ii) follows from Theorem 3.1.

This shows that trying to extend [2, Corollary 3.4] from quadratic to diagonal forms of higher degree results in a much weaker version.

\section{2 .}

Let $K$ be a $p$-adic field with residue field $k$ such that $\operatorname{char}(k) \nmid d$ !.

Corollary 3.4. Any diagonal form of degree $d$ and dimension $>d^{3}+1$ over a function field in one variable $F=K(t)$ is 
(i) isotropic over $F_{v}$, for every discrete valuation $v$ with residue field either a function field in one variable over $k$ or a finite extension of $K$;

(ii) isotropic over $F_{U}$ for each reduced, irreducible component $U \subset Y$ of the complement of $S$ in $Y$ and isotropic over $F_{P}$ for each $P \in S$.

Proof. Every finite field $k$ is $C_{1}$ and so every diagonal form of degree $d$ and dimension $>d^{2}$ over any function field in one variable over $k$ (which is $C_{2}$ ) is isotropic. Assertion (i) is a direct consequence of Theorem 2.1 and (ii) follows from Corollary 3.3 (ii).

So if $\varphi$ is a diagonal form of degree $d$ in at least $d^{3}+1$ variables over $\mathbb{Q}(t)$ then $\varphi$ is isotropic over $\left(\mathbb{Q}_{p}(t)\right)_{U}$ for any $p \nmid d$ !, for each reduced, irreducible component $U \subset Y$ of the complement of $S$ in $Y$, and isotropic over $\left(\mathbb{Q}_{p}(t)\right)_{P}$ for each $P \in S$.

Remark 3.5. Let us compare Corollary 3.4 with the Ax-Kochen-Ersov Transfer Theorem [1]: given a degree $d$, for almost all primes $p$, a form of degree $d$ over $\mathbb{Q}_{p}$ of dimension greater than or equal to $d^{2}+1$ is isotropic [G, (7.4)]. Moreover, for any form $\varphi$ of degree $d \geq 2$ and dimension greater than $d^{3}$ over $\mathbb{Q}(t)$, for almost all primes $p$ the form $\varphi$ is isotropic over $\mathbb{Q}_{p}(t)$ ([16] for $d=2$, [12] for $\left.d \geq 3\right)$. The model-theoretic proofs of both results do not allow for a more concrete observation on which primes exactly are included here, nor can they be extended to other base fields.

Stronger upper bounds on $u_{\text {diag }}\left(d, \mathbb{F}_{q}(t)\right)$ will yield stronger results on its dimension, since we only used the upper bound in the well known inequality $d \cdot u_{\text {diag }}\left(d, \mathbb{F}_{q}\right) \leq u_{\text {diag }}\left(d, \mathbb{F}_{q}(t)\right) \leq d^{2}$ to prove Corollary 3.4, for instance we obtain:

Corollary 3.6. Assume that $u_{\text {diag }}(d, k(t))=d r<d^{2}$ for some $r \in\{1, \ldots, d-1\}$. Let $\varphi$ be a diagonal form of degree $d$ and dimension $>d^{2} r+1$ over a function field in one variable $F=K(t)$. Then:

(i) $\varphi$ is isotropic over $F_{v}$, for every discrete valuation $v$ with residue field either a function field in one variable over $k$ or a finite extension of $K$;

(ii) $\varphi$ is isotropic over $F_{U}$ for each reduced, irreducible component $U \subset Y$ of the complement of $S$ in $Y$ and over $F_{P}$ for each $P \in S$.

It is well known that $u_{\text {diag }}(d, K) \leq d^{2}$ for a $p$-adic field $K$ with residue field $k=\mathbb{F}_{q}$ [3]. Indeed, $u_{\text {diag }}(d, K)=d u_{\text {diag }}\left(d, \mathbb{F}_{q}\right)$ by Theorem 2.1, assuming that char $\mathbb{F}_{q}=p \nmid d$ ! as before, which shows that clearly $u_{\text {diag }}(d, K)$ can be smaller than $d^{2}$. On the other hand, Artin's conjecture that $\mathbb{Q}_{p}$ is a $C_{2}$-field is false for instance for forms of degree 4.

\section{Bibliography}

[1] J. Ax and S. Kochen, Diophantine problems over local fields I. Amer. J. Math. 87 (1965) 605-630. 
[2] J.-L. Colliot-Thélène, R. Parimala and V. Suresh, Patching and local global principles for homogeneous spaces over function fields of $p$-adic curves. Comment. Math. Helv. 87 (2012) (4), 1011-1033.

[3] H. Davenport and D. J. Lewis, Homogeneous additive equations. Proc. Royal Soc. Ser. A 272(1963), 443-460.

[G] M. J. Greenberg, Lectures on forms in many variables. W.A. Benjamin, Inc., New York, Amsterdam, 1969.

[4] D. Harbater and J. Hartmann, Patching over fields. Israel J. Math. 176 (2010), 61-107.

[5] D. Harbater, J. Hartmann and D. Krashen, Applications of patching to quadratic forms and central simple algebras. Invent. Math. 178 (2) (2009), 231-263.

[6] D. K. Harrison, A Grothendieck ring of higher degree forms. J. Algebra 35 (1975), 123-138.

[7] D. K. Harrison and B. Pareigis, Witt rings of higher degree forms. Comm. Alg. 16 (6) (1988), 1275-1313.

[8] S. Lang, Algebra. Third Edition. Addison-Wesley Publ. Comp. 1997.

[9] P. Morandi, Springer's theorem for higher degree forms. Math. Z. 256 (1) (2007), 221-228.

[10] M. Orzech, Forms of low degree in finite fields. Bull. Austral. Math. Soc. 30 (1) (1984), 45-58.

[11] R. Parimala and V. Suresh, Isotropy of quadratic forms over function fields of p-adic curves. Inst. Hautes Etudes Sci. Publ. Math. 88 (1998), 129-150.

[12] S. Pumplün, u-invariants for forms of higher degree. Expo. Math. 27 (2009), 37-53.

[13] J. E. Schneider, Orthogonal groups of nonsingular forms of higher degree. J. Alg. 27 (1973), 112-116.

[14] J.-P. Serre, Cohomologie Galoisienne. Fourth edition. Lecture Notes in Math. 5, Springer-Verlag, Berlin, Heidelberg and New York, 1973.

[15] T. A. Springer, Quadratic forms over a field with a discrete valuation. Indag. Math. 17 (1979), 33-39.

[16] K. Zahidi, On the u-invariant of p-adic function fields. Comm. Alg. 33 (7) (2005) $2307-2314$. 